\title{
Myelodysplastic Syndromes: What Do Hospitalists Need to Know?
}

\author{
Amer M. Zeidan, MD, Bishoy Faltas, MD², B. Douglas Smith, MD¹, Steven Gore, MD¹
}

${ }^{1}$ Department of Oncology, The Sidney Kimmel Comprehensive Cancer Center at Johns Hopkins University, Baltimore, Maryland; ${ }^{2}$ Division of Hematology and Medical Oncology, Weill-Cornell Medical College, New York, New York.

Myelodysplastic syndromes (MDS) comprise a heterogeneous group of clonal hematopoietic stem cell disorders characterized by dysplasia, ineffective hematopoiesis leading to peripheral blood cytopenias, and a variable risk of leukemic progression. MDS primarily affects the elderly, and although its prevalence is increasing, MDS is frequently overlooked and underdiagnosed. MDS should be suspected in any patient with unexplained cytopenias, and a bone marrow evaluation is ultimately needed to make the diagnosis and exclude other causes of bone marrow failure. The last 15 years have witnessed significant advances in our understanding of the complex pathogenesis, classification and prognostication, and therapeutic approaches to MDS. Several prognostic models facilitate outcome prediction and risk-adapted therapy. The addition of azacitidine, decitabine, and lenalidomide to erythropoiesis-stimulating agents in our armamentarium offered new effective therapeutic options for many patients who are not candidates for intensive interventions. Improved understanding of the genetic, epigenetic, and immunologic mechanisms that operate in MDS will help develop better prognostication tools and rationally design more effective therapies. Hospitalists are likely to encounter both patients with MDS and patients in whom MDS should be suspected. In this review of MDS, we focus on the epidemiology, diagnosis, pathogenesis, classification and prognostic tools, and treatment options. Journal of Hospital Medicine 2013;8:351-357. (C) 2013 Society of Hospital Medicine
Myelodysplastic syndromes (MDS) comprise a heterogeneous group of clonal hematopoietic stem cell neoplasms characterized by dysplasia, ineffective hematopoiesis resulting in peripheral blood (PB) cytopenias affecting one or more cell lines, and a variable risk of progression to acute myeloid leukemia (AML). The last 15 years have witnessed significant advances in our understanding of the complex pathogenesis, classification and prognostication, and therapeutic approaches to MDS. As more elderly patients are diagnosed with MDS, encounters with hospitalized MDS patients or patients in whom MDS should be considered in the differential diagnosis are common events for today's hospitalists. In this review, we discuss the epidemiology, diagnosis, pathogenesis, prognostication, and therapies for MDS, with an emphasis on practical aspects that would be useful for hospitalists caring for these patients.

\section{EPIDEMIOLOGY OF MDS}

Although MDS is one of the most common hematologic malignancies, MDS remains understudied epidemiologically. ${ }^{1,2}$ Our understanding of the epidemiology improved after the implementation of

*Address for correspondence and reprint requests: Amer Zeidan, MD, Department of Oncology, Sidney Kimmel Comprehensive Cancer Center at Johns Hopkins University, 1650 Orleans Street, CRB1 Building, Room 186, Baltimore, MD 21287; Telephone: 410-614-4459;

Fax: 410-955-0185; E-mail: azeidan1@jhmi.edu

Additional Supporting Information may be found in the online version of this article.

Received: January 30, 2013; Revised: March 29, 2013; Accepted: April 1, 2013

2013 Society of Hospital Medicine DOI 10.1002/jhm.2049

Published online in Wiley Online Library (Wileyonlinelibrary.com). reporting requirements to cancer registries, especially the Surveillance, Epidemiology, and End Results (SEER) database in 2001. ${ }^{1-3}$ Age-adjusted incidence of MDS in the United States ranged between 3.3 to 4.6 per 100,000 persons per year in the period between 2001 and 2008.,2,4 The majority of MDS patients are elderly, and because MDS incidence increases with age, the number of patients diagnosed with MDS is expected to continue to rise with the aging population. ${ }^{1,2,5}$ MDS is more common in men compared to women, and in Caucasians compared to African Americans. ${ }^{1,2}$ Different estimates put MDS prevalence in the United States somewhere between 60,000 and 170,000 persons. $^{2,6}$

\section{DIAGNOSIS OF MDS}

Many patients with MDS are asymptomatic at diagnosis and only come to medical attention due to abnormal blood counts done routinely or for other reasons. This contributes to MDS being underdiagnosed. When cytopenias are not severe enough to cause symptoms, it is also frequently overlooked in patients with mild anemia or other cytopenias. ${ }^{7}$ Together, being asymptomatic and having relatively mild cytopenias are probably the most important factors that lead to under-recognition of MDS among primary care physicians (PCPs). ${ }^{7-9}$ There is a misconception that anemia is normal in the elderly, and when patients are not symptomatic that a workup is not needed. ${ }^{6,7}$ This is compounded by a lack of awareness of the importance of making a diagnosis in these patients and of currently available therapies for MDS. ${ }^{7-9}$

Anemia is not a normal consequence of aging and is always a pathologic state with an underlying 
etiology. ${ }^{6,7}$ Because a significant number of elderly patients with unexplained anemia could have MDS, patients with symptomatic or progressive anemia, especially if associated with other cytopenias, should be considered for further evaluation. ${ }^{7,9}$ Diagnosis is important given the recent availability of effective therapies for MDS that can improve anemia, decrease transfusion needs, improve life quality, and potentially increase survival. MDS is generally an indolent disease with a relative stability of blood counts in comparison to AML, so prior blood counts and the tempo of the process is an important consideration. ${ }^{9,10}$ The National Comprehensive Cancer Network clinical practice guidelines recommend exclusion of nutritional deficiencies (iron, vitamin B12, folate) and other causes of anemia (eg, gastrointestinal bleeding, renal insufficiency, and anemia of inflammation), assessment of reticulocyte count and serum erythropoietin level, and evaluation of a PB smear for evidence of dysplasia as important initial steps. ${ }^{10,11}$ Eventually the diagnosis of MDS requires a bone marrow (BM) evaluation to confirm the diagnosis and exclude other BM failure states by evaluating for BM cellularity, cell maturation, dysplasia (which should be present in at least $10 \%$ of any the myeloid lineages), percentage of blasts $(<20 \%)$, iron stores and sideroblasts, cytogenetics, MDS-specific fluorescence in situ hybridization (FISH) panels, flow cytometry, and other special testing., ${ }^{9,10}$ Despite extensive testing, MDS can sometimes be very difficult to differentiate from other bone marrow failure states (eg, hypoplastic MDS from aplastic anemia) (Table 1). ${ }^{10,11}$ In the absence of significant morbidity related to MDS, the definitive diagnosis of MDS can be usually made on an outpatient basis. It is important to ensure adequate follow-up with PCPs postdischarge and/or outpatient hematologist referral for patients with unexplained cytopenias.

\section{PATHOGENESIS AND ETIOLOGY OF MDS}

Ineffective hematopoiesis due to excessive apoptosis of hematopoietic precursors is a prominent feature of MDS, which explains the apparent paradox of hypercellular BM and PB cytopenias. Although not fully understood, complex epigenetic, genetic, and immunologic mechanisms contribute to the pathogenesis of MDS and account for disease heterogeneity. Aberrant silencing of tumor-suppressor and DNA repair genes mediated by hypermethylation of their promoters is believed to play an important part in the pathogenesis of MDS. ${ }^{12}$ This theory is supported by the unique sensitivity of MDS to drugs that reverse DNA methylation. Genetic abnormalities not only contribute to the pathogenesis of MDS, but are also among the strongest prognostic indicators for MDS patients, and can also affect therapeutic decisions. Clonal karyotypic abnormalities are observed in $50 \%$ of patients with MDS using conventional karyotyping. ${ }^{12,13}$ The most common chromosomal aberrations in MDS include
TABLE 1. Some of the Diseases That Should Be Differentiated From MDS and Their Main Differentiating Features

Idiopathic cytopenia of undetermined significance: no significant dysplasia or MDS-associated karyotypic aberrations

Acute myeloid leukemia: BM blasts $\geq 20 \%$, presence of core-binding characteristic cytogenetic aberrations: t(8;21), t(15;17), inv(16) defines AML regardless of BM blast count; AML can be associated with hepatosplenomegaly or myeloid sarcomas

Chronic myeloid leukemia: presence of Philadelphia chromosome t(9;22) positive, basophilia, and splenomegaly

Myelofibrosis: significant BM fibrosis, splenomegaly, and leukoerythroblastic picture in PB (teardrop and nucleated RBCs, left-shifted myeloid cells)

Chronic myelomonocytic leukemia: significant PB monocytosis

MDS/MPN overlap syndromes: dysplasia with myeloproliferative characteristics such as splenomegaly, thrombocytosis, or leukocytosis

Infections: for example, HIV and parvovirus B19 infections

Myelophthisis: infiltration of BM with other tumors (eg, melanoma) with resultant PB cytopenias

Nutritional disturbances: B12, folate, and copper deficiency, and zinc and arsenic excess can mimic MDS

Medications: drugs that interfere with DNA synthesis such as HIV medications, chemotherapeutic agents, cotrimoxazole, methotrexate, azathioprine, and G-CSF

Immune disorders: for example, LGL leukemia, lupus, or rheumatoid arthritis

Other acquired or congenital hematological disorders: for example, paroxysmal nocturnal hemoglobinuria, congenital dyserythropoietic anemia, dyskeratosis congenita

NOTE: Abbreviations: AML, acute myeloid leukemia; BM, bone marrow; G-CSF, colony granulocyte-stimulating factor ${ }^{11,21}$; HIV, human immunodeficiency virus; LGL, large granular lymphocytic; MDS, myelodysplastic syndromes; MPN, myeloproliferative disorders; PB, peripheral blood; RBCs, red blood cells.

deletions of the long arm of chromosome 5 (del5q), monosomy Y, monosomy 7 (del7) or deletion of its long arm (del7q), trisomy 8, del20q, and complex karyotypes ( $\geq 3$ chromosomal aberrations). ${ }^{12,13}$ These cytogenetic abnormalities correlate with the prognosis of MDS (eg, poor prognosis with complex karyotypes and chromosome 7 deletions vs better prognosis with isolated del $5 \mathrm{q}) .{ }^{12,13}$

Recently, FISH assays and genome-wide screening techniques (eg, single nucleotide polymorphism arrays, array-based comparative genomic hybridization, whole genome or exome sequencing) have enabled detection of an increasing number of genetic aberrations and recurrent somatic molecular abnormalities in a significant number of MDS patients (eg, abnormalities of ASXL1, IDH1/IDH2, DNMT3, EZH2, TET2, and SF3B1 genes). ${ }^{12,14}$ Most affected genes are involved in the epigenetic regulation of transcription (DNA methylation and demethylation, histone posttranslational modification) or mRNA splicing. ${ }^{12-14}$

Immunologic aberrations have also been proposed to contribute to pathogenesis of MDS. For example, in early-stage MDS, an aberrant immune attack on myeloid progenitors resulting in increased apoptosis can contribute to BM failure. ${ }^{15}$ This is supported by association of some forms of MDS with autoimmune diseases and observed responses in some patients to immunosuppressive therapies. The relative contribution of pathogenetic mechanisms varies between the different MDS subtypes. For example, haploinsufficiency of cell-cycle regulatory and ribosomal protein 


\begin{tabular}{|c|c|c|}
\hline MDS WHO Class & PB Findings & BM Findings \\
\hline $\begin{array}{l}\text { Refractory cytopenias with unilineage dysplasia: includes } \\
\text { refractory anemia; refractory neutropenia; refractory } \\
\text { thrombocytopenia }\end{array}$ & Unicytopenia or bicytopenia; PB blasts $<1 \%$ & $\begin{array}{l}\text { BM blasts }<5 \% \text {; unilineage dysplasia ( } \geq 10 \% \text { of cells in any } \\
\text { myeloid lineage); }<15 \% \text { of enythroid precursors are } \\
\text { ringed sideroblasts }\end{array}$ \\
\hline Refractory anemia with ring sideroblasts & Anemia; PB blasts $<1 \%$ & $\begin{array}{l}\text { BM blasts }<5 \% \text {; erythroid dysplasia only; } \geq 15 \% \text { of enthroid } \\
\text { precursors are ringed sideroblasts }\end{array}$ \\
\hline Refractory cytopenia with multilineage dysplasia & Cytopenia(s); PB blasts $<1 \%$; no Auer rods; $<1 \quad \times 10^{6} / \mu \mathrm{L}$ monocytes & $\begin{array}{l}\text { BM blasts }<5 \% \text {; dysplasia }(\geq 10 \% \text { of cells in at least } 2 \\
\text { myeloid lineages); no Auer rods }\end{array}$ \\
\hline Refractory anemia with excess blasts-1 & Cytopenia(s); PB blasts <5\%; no Auer rods; $<1 \quad \times 10^{6} / \mu \mathrm{L}$ monocytes & $\begin{array}{l}\text { BM blasts 5\%-9\%; unilineage or multilineage dysplasia; no } \\
\text { Auer rods }\end{array}$ \\
\hline Refractory anemia with excess blasts-2 & Cytopenia(s); PB blasts 5\%-19\%; Auer rods; $<1 \times 10^{6} / \mu \mathrm{L}$ monocytes & $\begin{array}{l}\text { BM blasts 10\%-19\%; unilineage or multilineage dysplasia; } \\
\text { Auer rods }\end{array}$ \\
\hline Myelodysplastic syndrome-unclassified & Cytopenias; PB blasts $\leq 1 \%$ & $\begin{array}{l}\text { BM blasts }<5 \% \text {; unequivocal dysplasia in }<10 \% \text { of cells at } \\
\text { least one myeloid cell lines when accompanied by a } \\
\text { cytogenetic abnormality considered as presumptive } \\
\text { evidence for a diagnosis of MDS }\end{array}$ \\
\hline MDS associated with isolated del5q & Anemia; normal to elevated platelet count; PB blasts $<1 \%$ & $\begin{array}{l}\text { BM blasts <5\%; normal to elevated megakaryocytes with } \\
\text { hypolobated nuclei; ;solated del5q karyotypic abnormality; } \\
\text { no Auer rods }\end{array}$ \\
\hline
\end{tabular}

NOTE: Dysplasia has to be present in at least $10 \%$ of the cells in the affected myeloid lineage. Cytopenias are defined as: neutropenia, absolute neutrophil count < $1800 / \mu \mathrm{L} ;$ anemia, hemoglobin < $10 \mathrm{~g} / \mathrm{dL}$; and thrombocytopenia, platelet count $<100,000 / \mu \mathrm{L}$. Adapted from Swerdlow et al. ${ }^{21}$

Abbreviations: BM, bone marrow; MDS, myelodysplastic syndromes; PB, peripheral blood; WHO, World Health Organization.

genes located in the commonly deleted region of $5 \mathrm{q}$ play an important role in the pathogenesis of MDS with isolated del5q $\left(5 \mathrm{q}-\right.$ syndrome). ${ }^{16}$ Mutations in the RNA spliceosomal machinery gene $S F 3 B$ have been shown to play a role in the pathogenesis of the MDS subtype refractory anemia with ringed sideroblasts (RARS), with those patients with RARS carrying this mutation having a more favorable prognosis than those with the wild-type gene. ${ }^{14}$ Several excellent recent reviews provide detailed discussion of the complex pathophysiology of MDS. ${ }^{12-14,17}$

Approximately $10 \%$ of MDS patients have secondary MDS (MDS occurring after chemotherapy or radiation therapy administration for treatment of another malignancy). ${ }^{2}$ Aside from advancing age, the causative factors for the other $90 \%$ of cases (primary MDS) are unknown in most patients, although environmental and occupational exposures (eg, smoking, painting, insecticides, pesticides, organic solvents), and genetic syndromes (eg, DNA repair defects such as Fanconi's anemia) are implicated in some patients. ${ }^{2,10}$ Recently, an epidemiologic study found an increased MDS risk with obesity. ${ }^{18}$

\section{PROGNOSTICATION OF MDS}

MDS is a form of cancer, and most affected patients eventually die from cytopenic complications or leukemic progression. MDS is not a single disease but rather encompasses a group of heterogeneous subtypes with significantly different natural histories and pace of progression. Therefore, accurate risk stratification of MDS is necessary not only to predict survival and risk of leukemic progression, but also to help choose the most appropriate therapeutic option for individual patients. Information about prognosis should also be utilized when making management decisions with patients for other comorbid conditions (eg, major surgery). Two morphologically based classification systems are commonly used for MDS: the FrenchAmerican-British (FAB) system and the World Health Organization (WHO) classification (Table 2), which most recently has supplanted the FAB system as the primary pathologic classification system. ${ }^{19-21}$ Several prognostic models have been developed around the morphologic classifications to better account for relevant clinical and cytogenetic modifiers of this disease. Although some of these models have been validated by different groups, each of these models has limitations. Although the predictions generated by these models are generally accurate for the different prognostic categories to which the patient is assigned, the extent to which the prediction applies to an individual MDS patient can vary significantly. In addition, comorbid conditions affect survival of MDS patients and are not included in the specific scoring systems. For example, congestive heart failure and chronic obstructive lung disease were associated with shortened survival in MDS patients. ${ }^{18}$

The International Prognostic Scoring System (IPSS) is the most widely used prognostic tool for MDS (Table 3). ${ }^{22}$ In this model, an aggregate score is calculated based on points assigned to the percentage of blasts in BM, the number of PB cell lines affected by cytopenias, and the karyotype. Based on this point score, the patient is assigned to 1 of 4 categories that portend significantly different outcomes: low, intermediate-1 (INT-1), intermediate-2 (INT-2), and high risk. The IPSS was developed from a database of mostly untreated MDS patients and does not account for other important prognostic parameters such as 
TABLE 3. International Prognostic Scoring System for Prognostication of MDS

Calculation of Score Value Based on Prognostic Variables

\begin{tabular}{|c|c|c|c|c|c|}
\hline & \multicolumn{5}{|c|}{ Score Value } \\
\hline & 0 & 0.5 & 1.0 & 1.5 & 2.0 \\
\hline $\begin{array}{l}\text { Prognostic variable } \\
\text { Bone marrow blasts }(\%)^{*} \\
\text { Karyotype }^{\dagger} \\
\text { Number of peripheral blood cell } \\
\text { line affected by cytopenias }{ }^{\ddagger}\end{array}$ & $\begin{array}{l}<5 \\
\text { Good } \\
0 \text { or } 1\end{array}$ & $\begin{array}{l}5-10 \\
\text { Intermediate } \\
2 \text { or } 3\end{array}$ & $\frac{-}{\text { Poor }}$ & $11-20$ & $21-30$ \\
\hline \multicolumn{6}{|c|}{ Median Survival and Risk of Progression to AML According to the IPSS Risk Category in Absence of Therapy } \\
\hline Overall Score & $\begin{array}{c}\text { Risk } \\
\text { Category }\end{array}$ & $\begin{array}{l}\text { Percentage in the } \\
\text { IPSS Population }\end{array}$ & $\begin{array}{c}\text { Median Survival } \\
\text { (Years) }\end{array}$ & \multicolumn{2}{|c|}{$\begin{array}{c}\text { Median Time From } \\
\text { Diagnosis at Which 25\% of } \\
\text { Patients Progress to AML (Years) }\end{array}$} \\
\hline 0 & Low & $33 \%$ & 5.7 & \multicolumn{2}{|c|}{9.4} \\
\hline $0.5-1.0$ & INT-1 & $38 \%$ & 3.5 & \multicolumn{2}{|c|}{3.3} \\
\hline $1.5-2.0$ & INT-2 & $22 \%$ & 1.1 & \multicolumn{2}{|c|}{1.1} \\
\hline$>2.5$ & High & $7 \%$ & 0.4 & \multicolumn{2}{|c|}{0.2} \\
\hline
\end{tabular}

NOTE: For therapeutic purposes, the IPSS low and INT-1 risk groups are considered lower-risk MDS, whereas the IPSS INT-2 and high-risk groups are considered higher-risk MDS. Adapted from Greenberg et al. ${ }^{22}$ Abbreviations: AML, acute myeloid leukemia; INT-1, intermediate-1; INT-2, intermediate-2; IPSS, International Prognostic Scoring System; MDS, myelodysplastic syndromes; WHO, World Health Organization.

*Patients with 21\%-30\% blasts are considered as AML according to WHO classification.

${ }^{\dagger}$ Cytogenetics: good = normal, isolated delY, isolated del5q, isolated del20q; Poor = complex ( $\geq 3$ abnormalities) or chromosome 7 anomalies; intermediate $=$ other abnormalities

${ }^{\ddagger}$ Cytopenias: neutrophil count, $<1800 / \mu \mathrm{L} ;$ platelets, $<100,000 / \mu \mathrm{L}$; hemoglobin $<10 \mathrm{~g} / \mathrm{dL}$.

transfusion dependence, depth of cytopenias, and extent/severity of lineage dysplasia. ${ }^{22}$ The WHO Prognostic Scoring System was proposed to overcome some of these shortcomings. ${ }^{23,24}$ Efforts to continue to improve the prognostic models further led to a large international collaboration that compiled a much larger database and resulted in the development of a revised IPSS (IPSS-R). ${ }^{25} \mathrm{New}$ discoveries of novel prognostic epigenetic, genetic, and immunologic determinants will likely result in the ongoing evolution of the current prognostic systems to further improve their discriminatory power. ${ }^{26}$

\section{MANAGEMENT OF MDS}

Most patients with MDS were treated historically with supportive measures only. The approval of 3 agents for treatment of MDS including the DNA methyltransferase inhibitors (DNMTi) azacitidine and decitabine, as well as the immunomodulatory agent lenalidomide, in the last decade advanced the care of MDS patients significantly (Table 4). Nonetheless, the use of allogeneic hematopoietic stem cell transplantation (alloHSCT) remains the only known curative modality for patients with MDS and should always be considered as a possible therapeutic option. ${ }^{27}$ Unfortunately, the majority of patients with MDS are not considered candidates for alloHSCT due to age, comorbidities, and lack of suitable donors. ${ }^{27}$ Therefore, most patients with MDS are managed with noncurative treatment and supportive paradigms. Treatment goals generally depend on the risk stratification for the particular individual, age, functional status, comorbidities, and importantly, the patient's individual preference. For medical decision-making purposes, MDS is traditionally divided into 2 major risk categories: low-risk (LR) and high-risk (HR) groups. LR-MDS includes the IPSS risk categories of low or INT-1, whereas HR-MDS is usually defined by the IPSS risk categories of INT-2 and high. Newer classification tools (eg, IPSS-R) and better molecular markers are expected to impact such categories as well as treatment recommendations in the future. ${ }^{26}$

Despite recent advances, supportive care for all patients with MDS remains a very important aspect of management, either in combination with other therapies or as sole therapy for frail patients who cannot tolerate further interventions. Supportive therapy focuses on maintaining a high quality of life and includes careful blood count monitoring, use of growth factors, use of transfusions and antibiotics as needed, and use of iron chelation therapy in some patients. Some of the common situations in which hospitalists encounter patients with MDS are listed in Table 5.

\section{MANAGEMENT OF LR-MDS}

In addition to supportive care or enrollment in clinical trials, therapies for LR-MDS include erythropoiesisstimulating agents, lenalidomide, and immunosuppressive therapy.

\section{Erythropoiesis-Stimulating Agents}

Anemia in MDS is a multifactorial process that includes ineffective erythropoiesis and suboptimal serum erythropoietin responses. ${ }^{10,28,29}$ There are no randomized studies to suggest that erythropoiesisstimulating agents (ESA) therapy prolongs survival in 


\section{TABLE 4. US Food and Drug Administration- Approved Drugs Used to Treat MDS}

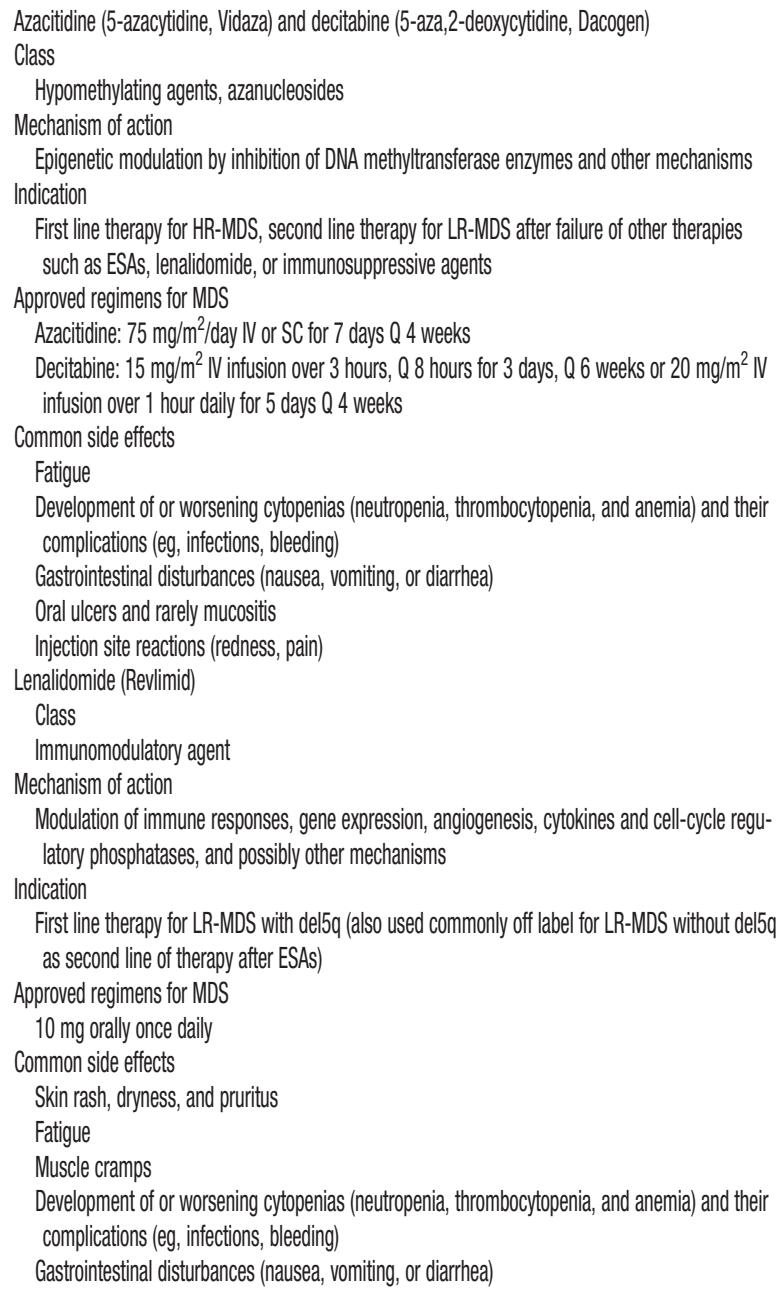

NOTE: Abbreviations: Del5q deletions in long arm of chromosome 5; ESAs, enthropoiesis-stimulating agents; HR, higher risk; IV, intravenous; LR, lower risk; MDS, myelodysplastic syndromes; Q, every; SC, subcutaneous.

MDS patients. Nonetheless, ESAs improve anemia significantly in some patients and are widely used. ${ }^{30,31}$ Approximately $20 \%$ to $30 \%$ of unselected MDS patients and about $40 \%$ of LR-MDS patients achieve clinically meaningful erythroid responses with ESA therapy with a median response duration of 2 years. ${ }^{30,31}$ It is important to correct coexisting nutritional deficiencies (eg, iron or folate deficiency) to optimize responses to ESA. ${ }^{10}$ Granulocyte colonystimulating factor can be synergistic with ESAs especially in patients with RARS. ${ }^{10}$ Patients with LR-MDS who have low endogenous serum erythropoietin levels $(<200-500 \mathrm{mU} / \mathrm{mL})$ and lower red blood cell (RBC) transfusion requirements $(<2 \mathrm{U}$ per month) are more likely to respond to ESA therapy. ${ }^{32,33}$ Compared to certain solid tumors, ESA therapy in MDS has not been associated with an increased risk of thromboembolic events. ${ }^{34}$
TABLE 5. Common Situations in Which Hospitalists Encounter Patients With MDS and Their Suggested Management

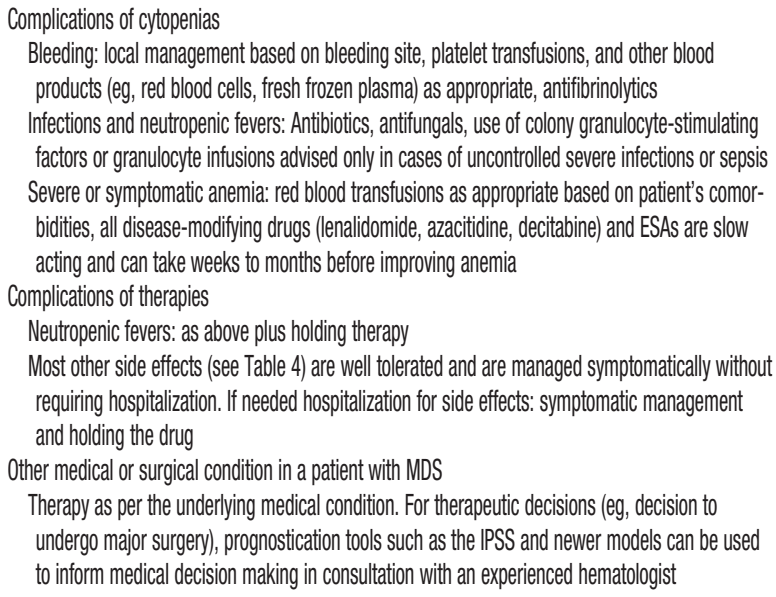

NOTE: These recommendations are general guidelines, and consultation with an experienced hematologist is recommended.

Abbreviations: ESAs, erythropoiesis-stimulating agents; IPSS, International Prognostic Scoring System; MDS, myelodysplastic syndromes.

\section{Lenalidomide}

$5 \mathrm{q}-$ syndrome is a subtype of MDS characterized by refractory macrocytic anemia, normal or elevated platelet counts, low BM blast percentage, small hypolobated dysplastic megakaryocytes, an isolated interstitial deletion in $5 \mathrm{q}$, and an indolent natural history. ${ }^{17,35}$ Lenalidomide, an oral derivative of thalidomide, induces high response rates in LR-MDS patients with $5 \mathrm{q}$ deletions, including hematologic improvements, RBC transfusion independence (TI) (56\%$67 \%$, median duration $>104$ weeks), cytogenetic responses $(50 \%-76 \%)$, and complete remissions. ${ }^{35,36}$ These findings resulted in approval of lenalidomide (Revlimid; Celgene Corp., Summit, NJ) for patients with IPSS low or INT-1 MDS with transfusion-dependent anemia and $5 \mathrm{q}$ deletions with or without additional cytogenetic abnormalities. In addition, lenalidomide has some activity against LR-MDS without $5 \mathrm{q}$ deletions (TI, $26 \%$, median duration 41 weeks) and some patients with HR-MDS and $5 \mathrm{q}$ deletions (TI, 25.5\%, median duration 26 weeks. ${ }^{37,38}$ Therefore, lenalidomide is a reasonable consideration in some patients with LR-MDS without $5 \mathrm{q}$ deletions with primary or secondary resistance to ESA therapy. ${ }^{10}$

\section{Immunosuppressive Therapy}

Some patients with LR-MDS respond to immunosuppressive therapy with antithymocyte globulin with or without cyclosporine. Characteristics that correlate with higher response rates: LR-MDS, younger age (<60 years), hypoplastic MDS, normal karyotype, human leukocyte antigen-DR15 histocompatibility type, and presence of a paroxysmal nocturnal hemoglobinuria clone. ${ }^{10,39}$ 


\section{MANAGEMENT OF HR-MDS}

The goal of management for HR-MDS is to modify the natural history of the disease and to prolong survival. In addition to a supportive care-only approach or clinical trial referral, 3 standard therapeutic approaches are used for patients with HR-MDS: alloHSCT, intensive chemotherapy, and DNMTi therapy. The use of intensive AML-like chemotherapy for HR-MDS is associated with high toxicity and very limited long-term success. Despite recent innovations in the field of transplantation, only a minority of MDS patients undergo alloHSCT, as most patients with HR-MDS are elderly and/or medically infirm. Even for the minority of patients who do undergo alloHSCT, relapse after alloHSCT remains a major challenge.

\section{DNA Methyltransferase Inhibitor Therapy}

5-azacitidine (AZA), (Vidaza; Celgene Corp.) and decitabine (DAC) (Dacogen; Eisai, Inc.) are potent inhibitors of DNA methyltransferases, which are enzymes responsible for cytosine methylation. ${ }^{38,40}$ These socalled differentiation agents appear to restore normal hematopoiesis for many MDS patients, and the approved regimens of DNMTi in MDS result in overall response rates in about $40 \%$ to $60 \%$ of patients. Unfortunately, complete remissions (CR) are rare $(10 \%-20 \%)$ and the duration of responses are also somewhat limited (median CR duration, 10 to 14 months). ${ }^{41-44}$ In randomized clinical trials, both AZA and DAC resulted in significant improvements in blood counts, reduction in transfusion needs, reduced infection rates, decreased risk of progression to AML, and improvements in patient-reported quality-of-life measures. ${ }^{41-44}$ AZA, but not DAC, prolonged survival in HR-MDS patients in a large randomized trial (median overall survival for the AZA group was 24.5 months compared to 15 months for a group of patients treated with 1 of 3 conventional care regimens). ${ }^{41-44}$ AZA and DAC have not been compared head to head in trials, but most experts recommend AZA for first-line use in HR-MDS based on its effect on survival. ${ }^{10}$

AZA and DAC have also been studied as treatments for patients with AML. These agents differ from traditional intensive chemotherapy, as both agents are commonly administered on an outpatient basis, and hematologic responses are generally expected after 4 to 6 cycles of treatment as compared to a single course of intensive cytarabine-based induction chemotherapy used to treat AML. ${ }^{45}$ Additionally, the impact on survival may not require the achievement of a CR based on the finding that MDS patients saw improved survival even in patients whose best responses were hematologic improvements. ${ }^{46}$ However, therapy with DNMTi is not curative, and patients are maintained on treatment as long as they are responding and not experiencing major side effects. Still, all patients will eventually lose response to DNMTi.

\section{CONCLUSIONS}

MDS is a form of cancer that largely affects elderly patients and leads to a BM failure state and increased risk of leukemic transformation. MDS is underdiagnosed and is frequently overlooked in the differential diagnosis of anemia in the elderly. DNMTi, lenalidomide, and ESA therapy offer effective therapeutic options for many MDS patients, including some considered too old or frail for intensive medical interventions. The use of prognostic models help physicians and patients better understand the common course of patients with MDS and facilitate tailoring of riskadapted therapy. It is expected that our improved understanding of the genetic, epigenetic, and immunologic mechanisms that operate in MDS will help develop better classification tools and rationally design effective new therapies.

\section{Acknowledgments}

The authors thank Dr. Balazs Zsenits (Medical Director of the Rochester General Hospitalist Group, Rochester General Hospital, Rochester, NY) for his critical review of the article.

Disclosures: Dr. Steven Gore owned stock in Celgene until November 2011, received research support from Celgene and Novartis, and consulted for Celgene. Drs. B. Douglas Smith, Amer Zeidan, and Bishoy Faltas have no relevant disclosures.

\section{References}

1. Ma X. Epidemiology of myelodysplastic syndromes. Am J Med. 2012;125:S2-S5.

2. Sekeres MA. Epidemiology, natural history, and practice patterns of patients with myelodysplastic syndromes in 2010. J Natl Compr Canc Netw. 2011;9:57-63.

3. Scott B. Myelodysplastic syndromes: increasing disease awareness. Introduction. Am J Med. 2012;125:S1.

4. Rollison, DE, Howlader, N, Smith, MT, et al. Epidemiology of myelodysplastic syndromes and chronic myeloproliferative disorders in the United States, 2001-2004, using data from the NAACCR and SEER programs. Blood. 2008;112:45-52.

5. Ma X, Does M, Raza A, Mayne ST. Myelodysplastic syndromes: incidence and survival in the United States. Cancer. 2007;109:15361542.

6. Guralnik JM, Eisenstaedt RS, Ferrucci L, Klein HG, Woodman RC. Prevalence of anemia in persons 65 years and older in the United States: evidence for a high rate of unexplained anemia. Blood. 2004;104:2263-2268.

7. Scott B. Myelodysplastic syndromes: increasing disease awareness. Discussion. Am J Med. 2012;125:S33-S34.

8. Khan AM. Why are myelodysplastic syndromes unrecognized and underdiagnosed? A primary care perspective. Am J Med. 2012;125:S15-S17.

9. Foran JM, Shammo JM. Clinical presentation, diagnosis, and prognosis of myelodysplastic syndromes. Am J Med. 2012;125:S6-S13.

10. Greenberg PL, Attar E, Bennett JM, et al. NCCN Clinical Practice Guidelines in Oncology: myelodysplastic syndromes. J Natl Compr Canc Netw. 2011;9:30-56.

11. Steensma DP. Dysplasia has A differential diagnosis: distinguishing genuine myelodysplastic syndromes (MDS) from mimics, imitators, copycats and impostors. Curr Hematol Malig Rep. 2012;7:310-320.

12. Abdel-Wahab O, Figueroa ME. Interpreting new molecular genetics in myelodysplastic syndromes. Hematology Am Soc Hematol Educ Program. 2012;2012:56-64.

13. Tiu RV, Visconte V, Traina F, Schwandt A, Maciejewski JP. Updates in cytogenetics and molecular markers in MDS. Curr Hematol Malig Rep. 2011;6:126-135.

14. Visconte V, Makishima H, Maciejewski JP, Tiu RV. Emerging roles of the spliceosomal machinery in myelodysplastic syndromes and other hematological disorders. Leukemia. 2012;26:2447-2454. 
15. Epling-Burnette PK, Bai F, Painter JS, et al. Reduced natural killer (NK) function associated with high-risk myelodysplastic syndrome (MDS) and reduced expression of activating NK receptors. Blood. 2007;109:4816-4824.

16. Ebert BL, Pretz J, Bosco J, et al. Identification of RPS14 as a $5 \mathrm{q}-$ syndrome gene by RNA interference screen. Nature. 2008;451:335-339.

17. Bejar R, Levine R, Ebert BL. Unraveling the molecular pathophysiology of myelodysplastic syndromes. J Clin Oncol. 2011;29:504-515.

18. Ma X, Lim U, Park Y, et al. Obesity, lifestyle factors, and risk of myelodysplastic syndromes in a large US cohort. Am J Epidemiol. 2009;169:1492-1499.

19. Bennett JM, Catovsky D, Daniel MT, et al. Proposals for the classification of the myelodysplastic syndromes. $\mathrm{Br} J$ Haematol. 1982;51:189-199.

20. Harris NL, Jaffe ES, Diebold J, et al. World Health Organization classification of neoplastic diseases of the hematopoietic and lymphoid tissues: report of the Clinical Advisory Committee Meeting-Airlie House, Virginia, November 1997. J Clin Oncol. 1999;17:3835-3849.

21. Swerdlow SH, Campo E, Harris NL, et al. WHO classification of MDS. In: World Health Organization Classification of Tumours of Haematopoietic and Lymphoid Tissues. Lyon, France: IARC Press; 2008.

22. Greenberg P, Cox C, LeBeau MM, et al. International scoring system for evaluating prognosis in myelodysplastic syndromes. Blood. 1997;89:2079-2088.

23. Malcovati L, Germing U, Kuendgen A, et al. Time-dependent prognostic scoring system for predicting survival and leukemic evolution in myelodysplastic syndromes. J Clin Oncol. 2007;25:3503-3510.

24. Malcovati L, Della Porta MG, Strupp C, et al. Impact of the degree of anemia on the outcome of patients with myelodysplastic syndrome and its integration into the WHO classification-based Prognostic Scoring System (WPSS). Haematologica. 2011;96:1433-1440.

25. Greenberg PL, Tuechler H, Schanz J, et al. Revised international prognostic scoring system for myelodysplastic syndromes. Blood. 2012;120:2454-2465.

26. Zeidan AM, Smith BD, Komrokji RS, Gore SD. Prognostication in myelodysplastic syndromes: beyond the International Prognostic Scoring System (IPSS). Am J Med. 2013;126:e25.

27. Mufti GJ, Potter V. Myelodysplastic syndromes: who and when in the course of disease to transplant. Hematology Am Soc Hematol Educ Program. 2012;2012:49-55.

28. Parker JE, Fishlock KL, Mijovic A, Czepulkowski B, Pagliuca A, Mufti GJ. "Low-risk" myelodysplastic syndrome is associated with excessive apoptosis and an increased ratio of pro- versus anti-apoptotic bcl-2-related proteins. Br J Haematol. 1998;103:1075-1082.

29. Parker JE, Mufti GJ. Ineffective haemopoiesis and apoptosis in myelodysplastic syndromes. Br J Haematol. 1998;101:220-230.

30. Steensma DP. Hematopoietic growth factors in myelodysplastic syndromes. Semin Oncol. 2011;38:635-647.

31. Davidoff AJ, Weiss Smith S, Baer MR, et al. Patient and physician characteristics associated with erythropoiesis-stimulating agent use in patients with myelodysplastic syndromes. Haematologica. 2012;97: 128-132.
32. Park S, Grabar S, Kelaidi C, et al. Predictive factors of response and survival in myelodysplastic syndrome treated with erythropoietin and G-CSF: the GFM experience. Blood. 2008;111:574-582.

33. Hellstrom-Lindberg E, Negrin R, Stein R, et al. Erythroid response to treatment with G-CSF plus erythropoietin for the anaemia of patients with myelodysplastic syndromes: proposal for a predictive model. $\mathrm{Br} \mathrm{J}$ Haematol. 1997;99:344-351.

34. Smith SW, Sato M, Gore SD, et al. Erythropoiesis-stimulating agents are not associated with increased risk of thrombosis in patients with myelodysplastic syndromes. Haematologica. 2012;97:15-20.

35. List A, Dewald G, Bennett J, et al. Lenalidomide in the myelodysplastic syndrome with chromosome $5 \mathrm{q}$ deletion. $N$ Engl J Med. 2006;355:1456-1465.

36. Fenaux P, Giagounidis A, Selleslag D, et al. A randomized phase 3 study of lenalidomide versus placebo in RBC transfusion-dependent patients with low-/-ntermediate-1-risk myelodysplastic syndromes with del5q. Blood. 2011;118:3765-3776.

37. Raza A, Reeves JA, Feldman EJ, et al. Phase 2 study of lenalidomide in transfusion-dependent, low-risk, and intermediate- 1 risk myelodysplastic syndromes with karyotypes other than deletion $5 \mathrm{q}$. Blood. 2008;111:86-93.

38. Ades L, Boehrer S, Prebet T, et al. Efficacy and safety of lenalidomide in intermediate- 2 or high-risk myelodysplastic syndromes with $5 \mathrm{q}$ deletion: results of a phase 2 study. Blood. 2009;113:3947-3952.

39. Sloand EM, Wu CO, Greenberg P, Young N, Barrett J. Factors affecting response and survival in patients with myelodysplasia treated with immunosuppressive therapy. J Clin Oncol. 2008;26:2505-2511.

40. Griffiths EA, Gore SD. DNA methyltransferase and histone deacetylase inhibitors in the treatment of myelodysplastic syndromes. Semin Hematol. 2008;45:23-30.

41. Fenaux P, Mufti GJ, Hellstrom-Lindberg E, et al. Efficacy of azacitidine compared with that of conventional care regimens in the treatment of higher-risk myelodysplastic syndromes: a randomised, openlabel, phase III study. Lancet Oncol. 2009;10:223-232.

42. Silverman LR, Demakos EP, Peterson BL, et al. Randomized controlled trial of azacitidine in patients with the myelodysplastic syndrome: a study of the cancer and leukemia group B. J Clin Oncol. 2002;20:2429-2440.

43. Kantarjian H, Issa JP, Rosenfeld CS, et al. Decitabine improves patient outcomes in myelodysplastic syndromes: results of a phase III randomized study. Cancer. 2006;106:1794-1803.

44. Lubbert M, Suciu S, Baila L, et al. Low-dose decitabine versus best supportive care in elderly patients with intermediate- or high-risk myelodysplastic syndrome (MDS) ineligible for intensive chemotherapy: final results of the randomized phase III study of the European Organisation for Research and Treatment of Cancer Leukemia Group and the German MDS Study Group. J Clin Oncol. 2011;29:19871996.

45. Silverman LR, McKenzie DR, Peterson BL, et al. Further analysis of trials with azacitidine in patients with myelodysplastic syndrome: studies 8421,8921 , and 9221 by the Cancer and Leukemia Group B. J Clin Oncol. 2006;24:3895-3903.

46. Itzykson R, Thepot S, Quesnel B, et al. Prognostic factors for response and overall survival in 282 patients with higher-risk myelodysplastic syndromes treated with azacitidine. Blood. 2011;117:403-411. 